\title{
RESEARCH
}

Open Access

\section{Clinical utility of chitotriosidase enzyme activity in nephropathic cystinosis}

\author{
Mohamed A Elmonem?', Samuel H Makar ${ }^{2,3}$, Lambertus van den Heuvel ${ }^{4,5}$, Hanan Abdelaziz ${ }^{2,3}$, \\ Safaa M Abdelrahman ${ }^{2,3}$, Xavier Bossuyt ${ }^{6}$, Mirian C Janssen $^{7}$, Elisabeth AM Cornelissen ${ }^{5}$, Dirk J Lefeber ${ }^{8}$, \\ Leo AB Joosten ${ }^{5}$, Marwa M Nabhan ${ }^{2,3}$, Fanny O Arcolino ${ }^{4}$, Fayza A Hassan ${ }^{1}$, Héloïse P Gaide Chevronnay ${ }^{9}$, \\ Neveen A Soliman ${ }^{2,3}$ and Elena Levtchenko ${ }^{4^{*}}$
}

\begin{abstract}
Background: Nephropathic cystinosis is an inherited autosomal recessive lysosomal storage disorder characterized by the pathological accumulation and crystallization of cystine inside different cell types. WBC cystine determination forms the basis for the diagnosis and therapeutic monitoring with the cystine depleting drug (cysteamine). The chitotriosidase enzyme is a human chitinase, produced by activated macrophages. Its elevation is documented in several lysosomal storage disorders. Although, about $6 \%$ of Caucasians have enzyme deficiency due to homozygosity of 24-bp duplication mutation in the chitotriosidase gene, it is currently established as a screening marker and therapeutic monitor for Gaucher's disease.

Methods: Plasma chitotriosidase activity was measured in 45 cystinotic patients, and compared with 87 healthy controls and 54 renal disease patients with different degrees of renal failure (CKD1-5). Chitotriosidase levels were also correlated with WBC cystine in 32 treated patients. Furthermore, we incubated control human macrophages in-vitro with different concentrations of cystine crystals and monitored the response of tumor necrosis factor-alpha (TNF-a) and chitotriosidase activity. We also compared plasma chitotriosidase activity in cystinotic knocked-out $(n=10)$ versus wild-type mice $(n=10)$.
\end{abstract}

Results: Plasma chitotriosidase activity in cystinotic patients (0-3880, median $163 \mathrm{nmol} / \mathrm{ml} / \mathrm{h}$ ) was significantly elevated compared to healthy controls (0-90, median $18 \mathrm{nmol} / \mathrm{ml} / \mathrm{h})$ and to CKD patients $(0-321$, median $52 \mathrm{nmol} / \mathrm{ml} / \mathrm{h})$, $\mathrm{P}<0.001$ for both groups. Controls with decreased renal function had mild to moderate chitotriosidase elevations; however, their levels were significantly lower than in cystinotic patients with comparable degree of renal insufficiency. Chitotriosidase activity positively correlated with WBC cystine content for patients on cysteamine therapy $(r=0.8)$, $P<0.001$. In culture, human control macrophages engulfed cystine crystals and released TNF-a into culture supernatant in a crystal concentration dependent manner. Chitotriosidase activity was also significantly increased in macrophage supernatant and cell-lysate. Furthermore, chitotriosidase activity was significantly higher in cystinotic knocked-out than in the wild-type mice, $P=0.003$.

Conclusions: This study indicates that cystine crystals are potent activators of human macrophages and that chitotriosidase activity is a useful marker for this activation and a promising clinical biomarker and therapeutic monitor for nephropathic cystinosis.

Keywords: Lysosomal storage disorders, Nephropathic cystinosis, Cystine crystals, Macrophage activation, Chitotriosidase enzyme, Clinical screening, Cysteamine, Therapeutic monitoring

\footnotetext{
* Correspondence: elena.levtchenko@uzleuven.be

${ }^{4}$ Department of Pediatric Nephrology \& Growth and Regeneration, University

Hospital Leuven, Catholic University of Leuven, Leuven, Belgium

Full list of author information is available at the end of the article
} 


\section{Background}

Nephropathic cystinosis (NC) is the prototype disorder for lysosomal transporter protein defects. CTNS gene mutations cause deficiency of cystinosin, the protein that transports cystine out of the lysosomes, resulting in the accumulation and crystallization of cystine in virtually all body cells. NC patients are asymptomatic at birth and usually develop normally during the first six months of life, where after they start to present with renal Fanconi syndrome. If not treated, renal failure invariably develops within the first decade of life [1].

The aminothiol cysteamine is the only specific treatment known today. It depletes lysosomal cystine through the formation of cysteine and the mixed disulfide cysteaminecysteine complex which exits the lysosome via an intact PQLC2-transporter [2]. Cysteamine slows down the deterioration of the kidney function and postpones or even prevents extra-renal damage [1].

Elevated white blood cell (WBC) cystine concentration is the diagnostic cornerstone, as the pathognomonic corneal cystine crystals can frequently be missed in children younger than 18 months and genetic testing usually takes a longer time [3]. WBC cystine assay was introduced by Oshima et al. who used $\left[{ }^{14} \mathrm{C}\right]$ cystine and E.coli cystinebinding protein [4]. Currently most laboratories switched to HPLC [5] or LC-MS/MS [6] methods to avoid working with radioactivity. While biochemical cystine determination can be well-standardized, the major source of imprecision is attributed to the WBC isolation and storage [7]. Furthermore, blood for cystine assay must be taken six hours after the last cysteamine dose which is not always clinically feasible. Finally, it is currently unknown whether WBC cystine levels adequately represent cystine accumulation in tissues.

Chitotriosidase was first associated with marked lysosomal storage in Gaucher's disease patients [8]. Later its elevation was detected in many other lysosomal storage disorders (LSDs) [9] and also in multiple other diseases as $\beta$-thalassemia [10] and sarcoidosis [11].

Due to the absence of its natural substrate (chitin) in mammals and its secretion mainly by activated macrophages, chitotriosidase was rapidly linked to innate immunity as a defense mechanism against chitin coated pathogens [12]. However, its elevation in response to storage and chronic inflammatory disorders clarified its other nature as a marker of macrophage activation.

Despite the fact that about $6 \%$ of Caucasians have complete enzyme deficiency due to homozygosity to the 24-bp duplication mutation at exon 10 of the chitotriosidase gene (CHIT1) [13], plasma chitotriosidase activity has been established as a screening marker and therapeutic monitor for enzyme replacement therapy in Gaucher's disease [14]. Further studies reported the validity of the clinical use of chitotriosidase activity in the screening of other lysosomal disorders as Niemann-Pick disease and Gangliosidosis M1 [15] and for therapeutic monitoring of male Fabry patients [16]. Recently, chitotriosidase elevation was documented in a case-report of nephropathic cystinosis [17], and it was suggested that it could be of value in monitoring cysteamine therapy.

In the current study, we aimed to evaluate the potential of plasma chitotriosidase activity for the clinical screening and therapeutic monitoring of NC patients. As a proof of principle we tested our hypothesis on the cellular level in control human macrophages and in the plasma of the murine cystinotic model.

\section{Methods \\ Patients}

Forty five NC patients were recruited over the period: November 2010- April 2013. Twenty three Egyptian patients (10 m-16y, median 3.8y) were recruited at the Center of Pediatric Nephrology and Transplantation (CPNT), Cairo University Children's Hospital, Egypt and 22 European patients (2-49y, median 26.5y) were recruited at Pediatric Nephrology Clinics, University Hospital Leuven, K.U. Leuven, Belgium and Pediatric Nephrology and Internal Medicine departments, Radboud University Medical Center, Nijmegen, the Netherlands.

Nine Egyptian patients were newly-diagnosed (10-28 m), while the rest of Egyptian and all European patients were already on cysteamine therapy. Chitotriosidase activities in cystinotic patients were compared to 54 healthy pediatric controls (1-16y, median 4.8y), 33 healthy adult controls (22-48y, median 36y), 24 renal pediatric controls: 12 with renal Fanconi syndrome after excluding NC (2-5y, median $3 y$ ) with chronic kidney disease (CKD 1-4) and 12 with end stage renal disease (ESRD, CKD 5) due to a variety of other causes (2-12y, median 6y), 15 patients with diabetic nephropathy (CKD 1-4, 26-46y, median 32y) and 15 patients with ESRD (CKD 5, 19-58y, median 41y). The current study was approved by the Institutional Research and Ethical Committee and written informed consents were obtained from healthy controls and patients or legal guardians in case of minors.

\section{Ctns knocked-out mice}

Blood was collected from the retro-orbital plexus of ten C57BL/6 Ctns(-/-) mice (12-15 m, median $13 \mathrm{~m})$ and ten wild-type mice $(12-15 \mathrm{~m}$, median $12 \mathrm{~m})$ with heparinized capillaries. Blood was centrifuged $20 \mathrm{~min}$ at $5000 \mathrm{rpm}$ and plasma was kept at $-80^{\circ} \mathrm{C}$ till assayed.

\section{L-cystine crystal preparation}

$120 \mathrm{mg}$ of L-cystine (Fluca) was dissolved in $200 \mathrm{ml}$ of distilled water through boiling while stirring. Solution was left to stand overnight. After centrifugation, supernatant was transferred to another container as fully saturated 
cystine solution, while the crystallized deposit was kept in $5 \mathrm{ml}$ of saturated solution. After vigorous mixing $0.5 \mathrm{ml}$ was transferred and dried in a vacuum centrifuge. According to the pellet's weight the crystal concentration was adjusted to $10 \mathrm{mg} / \mathrm{ml}$. Crystal size was mostly below $10 \mu \mathrm{m}$. Cystine saturated and crystal stock solutions were kept at $4^{\circ} \mathrm{C}$ till used.

\section{Monocyte maturation}

Heparinized blood was collected from healthy donors. Monocytes were separated with CD14 magnetic microbeads according to the supplier's protocol (MACS, Miltenyi Biotec) and incubated in RPMI-1640 (Lonza) supplemented with $10 \%$ FCS, $2 \mathrm{mM}$ L-glutamine and $50 \mu \mathrm{g} / \mathrm{ml}$ gentamycin. Cells were seeded at a concentration of 1-2million $/ \mathrm{ml}$, while adding $0.1 \mu \mathrm{g} / \mathrm{ml}$ GM-CSF (Gentaur) and $50 \mathrm{nmol} /$ $\mathrm{ml} \beta$-mercaptoethanol. After 3 days half amount of the culture medium was replaced with fresh medium containing GM-CSF $(0.2 \mu \mathrm{g} / \mathrm{ml})$ and $\beta$-mercaptoethanol $(50 \mathrm{nmol} /$ $\mathrm{ml})$. After another four days differentiated macrophages were harvested with trypsin $(170 \mathrm{U} / \mathrm{ml})+$ EDTA $(200 \mathrm{mg} / \mathrm{L})$ (Lonza) and immediately used.

\section{Macrophage exposure to cystine crystals}

Mature macrophages were seeded in $25 \mathrm{~cm}^{2}$ flasks at a density of 100,000 cells $/ \mathrm{ml}$, left to stand for 2 hours, then washed with PBS to eliminate non-adherent cells. Cystine crystals were washed three times in cystine saturated solution. Supernatant was discarded and cystine saturated culture medium was added (Culture stock solution with crystal concentration $10 \mathrm{mg} / \mathrm{ml})$, then two experiments were performed:

Experiment 1: There was no previous data available for the suitable amount of cystine crystals to stimulate macrophages, therefore we adapted a wide concentration range $\left(0-200 \mathrm{ug} / \mathrm{cm}^{2}\right)$ guided by similar experiments with other crystals as calcium oxalate [18]. Cystine crystal stock solution was mixed and transferred to fresh medium in concentrations ranging from 0 to $200 \mu \mathrm{g} / \mathrm{cm}^{2}$ $\left(0,1,5,25,100\right.$ and $\left.200 \mu \mathrm{g} / \mathrm{cm}^{2}\right)$, a triplicate of each concentration was performed in two separate experiments. Adherent macrophages were incubated with cystine crystals at $37^{\circ} \mathrm{C}, 5 \% \mathrm{CO} 2$ for three hours, and then washed with PBS to eliminate excess crystals and fresh medium was added. At determined intervals from the crystal elimination $(0,4,8,24$ and 48 hours) $300 \mu$ l of culture fluid was sampled, centrifuged and supernatant kept at $-80^{\circ} \mathrm{C}$ for TNF- $\alpha$ and chitotriosidase measurements. Resulting values were corrected for medium volume changes.
Experiment 2: Adherent macrophages were incubated with crystal solution at a fixed concentration of $100 \mu \mathrm{g} / \mathrm{cm}^{2}$, and then washed as previously described. At determined intervals: 0, 8, 24, 48 and 72 hours cells were harvested in duplicate. In the same experiment control macrophages were harvested at the same intervals. All pellets were kept immediately at $-80^{\circ} \mathrm{C}$ till assaying chitotriosidase activity.

\section{Microscopy}

Macrophages were visualized directly and after 48 hours by inverted phase contrast microscopy within culture flasks after incubation and washing excess crystals (DMIL-LED, Leica microsystems).

\section{TNF- $a$ immunoreactivity}

TNF- $\alpha$ was measured in culture supernatant by a sandwich ELISA technique according to the manufacturer's protocol (BD Pharmingen).

\section{Chitotriosidase enzyme activity}

Either plasma, culture supernatant or macrophage celllysate were used as samples. Macrophage lysis buffer included 1\% IGEPAL CA-630, 1\% SDS, 12 mM Na deoxycholate, $0.6 \mathrm{mM}$ PMSF, $1 \mu \mathrm{g} / \mathrm{ml}$ aprotinin and $1 \mathrm{mM} \mathrm{Na}$ orthovanadate (Sigma). Activity was measured as previously described [8]. Results were expressed as $\mathrm{nmol} / \mathrm{ml} / \mathrm{h}$ for plasma and nmol/mg pellet protein/h for supernatant and cell-lysate.

\section{Chitotriosidase genotype}

Being a common mutation in many ethnicities and the main cause of chitotriosidase deficiency worldwide [13], DNA analysis for detecting the 24-bp duplication mutation within exon ten of CHIT1 gene was performed for cystinotic patients. PCR was performed as previously described [13] resulting in the formation of a 75-bp band in the wild-type, a 99-bp band in the homozygously mutated and both in the heterozygous. Healthy controls for macrophage experiments were confirmed as being wildtype.

\section{WBC cystine assay}

Ten $\mathrm{ml}$ of blood was collected from NC patients. WBC were separated using dextran $3 \%$, and pellets were kept at $-80^{\circ} \mathrm{C}$ with $150 \mu \mathrm{l}$ of $\mathrm{N}$-ethylmaleimide and $50 \mu \mathrm{l}$ of $12 \%$ sulfosalicylic acid till assayed. Cystine was quantified by LC-MS/MS (Micromass, Waters) as previously described [6].

\section{Statistical analysis}

Unless otherwise stated all results were expressed as range and median. Differences were analyzed using a two-tailed 
Mann-Whitney U test with the Finner adjustment for multiple testing [WinPepi statistical software package]. Pearson correlation coefficient ( $r$ ) was used for testing correlations and $\mathrm{P}<0.05$ was set as the level of significance.

\section{Results}

\section{Plasma chitotriosidase activity}

Chitotriosidase levels in 45 cystinotic patients ranged from 0 to 3880 (median $163 \mathrm{nmol} / \mathrm{ml} / \mathrm{h}$ ), which were significantly different from 87 healthy controls ranging from 0 to 90 (median $18 \mathrm{nmol} / \mathrm{ml} / \mathrm{h}$ ), $\mathrm{P}<0.001$, including 54 healthy pediatric controls ranging from 0 to 72 (median $14 \mathrm{nmol} /$ $\mathrm{ml} / \mathrm{h}$ ) and 33 healthy adult controls ranging from 0 to 90 (median $31 \mathrm{nmol} / \mathrm{ml} / \mathrm{h}$ ). Levels in the cystinotic patients were also significantly different from the 54 CKD controls ranging from 0 to 321 (median $52 \mathrm{nmol} / \mathrm{ml} / \mathrm{h}$ ), $\mathrm{P}<0.001$. Renal controls consisted of 24 pediatric patients ranging from 2 to 144 (median $39 \mathrm{nmol} / \mathrm{ml} / \mathrm{h}$ ) and 30 ranging from
0 to 321 (median $73 \mathrm{nmol} / \mathrm{ml} / \mathrm{h}$ ). Table 1 summarizes chit otriosidase values for different subgroups of patients and controls. Poorly controlled cystinotic patients with ESRD demonstrated very high chitotriosidase values $(899,962$, 1346, 1487 and $3880 \mathrm{nmol} / \mathrm{ml} / \mathrm{h}), \mathrm{P}=0.002$ compared to the ESRD control group.

Among our series, nine cystinotic patients were newlydiagnosed. Their chitotriosidase activity ranged from 0 to 288 (median $122 \mathrm{nmol} / \mathrm{ml} / \mathrm{h}$ ), while their age matching healthy and CKD controls ranged from 0 to 49 (median $15 \mathrm{nmol} / \mathrm{ml} / \mathrm{h}$ ), $\mathrm{P}<0.001$ (Figure 1).

Chitotriosidase levels were generally higher in the 23 Egyptian patients ranging from 0 to 3880 (median $288 \mathrm{nmol} / \mathrm{ml} / \mathrm{h}$ ) compared to 22 European patients, in whom they ranged from 0 to 1487 (median $70 \mathrm{nmol} / \mathrm{ml} /$ h). On the other hand, WBC cystine levels in the ten measured Egyptian patients on treatment ranged from 1.5 to 15.3 (median $4.6 \mathrm{nmol} 1 / 2$ cystine/mg protein), while in the

Table 1 Chitotriosidase activity in control groups and cystinotic patients

\begin{tabular}{|c|c|c|c|c|}
\hline Group & $\mathbf{N}$ & $\begin{array}{l}\text { Age in years range } \\
\text { (median) }\end{array}$ & $\begin{array}{l}\text { Chitotriosidase activity } \mathrm{nmol} / \mathrm{ml} \\
\text { plasma/h range (median) }\end{array}$ & $P$ values \\
\hline All normal controls & 87 & $1-48(12)$ & $0-90(18)$ & \\
\hline Pediatric normal controls & 54 & $1-16(4.8)$ & $0-72(14)$ & \\
\hline Adult normal controls & 33 & $22-48(36)$ & $0-90(31)$ & $<0.001$ with pediatric normal controls \\
\hline All renal controls & 54 & $2-58(22)$ & $0-321(52)$ & $<0.001$ with all normal controls \\
\hline Pediatric renal controls & 24 & $2-12(4.6)$ & 2-144 (39) & $<0.001$ with pediatric normal controls \\
\hline Fanconi syndrome & 12 & $2-5(3)$ & $5-44(19)$ & 0.60 with pediatric normal controls \\
\hline \multirow[t]{2}{*}{ ESRD } & 12 & $2-12(6)$ & $2-144(67)$ & $<0.001$ with pediatric normal controls \\
\hline & & & & $<0.001$ with pediatric Fanconi syndrome \\
\hline Adult renal controls & 30 & $19-58(38)$ & $0-321(73)$ & $<0.001$ with adult normal controls \\
\hline Microalbuminuria & 15 & $26-46(32)$ & $8-143(60)$ & 0.008 with adult normal controls \\
\hline \multirow[t]{2}{*}{ ESRD } & 15 & $19-58(41)$ & $0-321(87)$ & $<0.001$ with adult normal controls \\
\hline & & & & 0.40 with diabetic nephropathy \\
\hline All controls with ESRD & 27 & $2-58(22)$ & $0-321(75)$ & \\
\hline All controls below 5 years & 39 & $0.5-4.5(2.2)$ & $0-49(15)$ & \\
\hline \multirow[t]{2}{*}{ Cystinosis patients } & 45 & $0.8-49(13)$ & $0-3880(163)$ & $<0.001$ with all normal controls \\
\hline & & & & $<0.001$ with all renal controls \\
\hline \multirow[t]{2}{*}{ All pediatric patients } & 25 & $0.8-16(3.8)$ & $0-3880(268)$ & $<0.001$ with pediatric normal controls \\
\hline & & & & $<0.001$ with pediatric renal controls \\
\hline \multirow[t]{2}{*}{ All adult patients } & 20 & $18-49(28)$ & $0-1487(78)$ & $<0.001$ with adult normal controls \\
\hline & & & & 0.44 with adult renal controls \\
\hline \multicolumn{5}{|l|}{ Cysteamine treated patients } \\
\hline Cystine $^{*}<1$ & 11 & $17-49(34)$ & $18-342(52)$ & 0.71 with all renal controls \\
\hline Cystine $^{*}>1$ and $<4$ & 13 & $2-38(24)$ & $0-843(245)$ & 0.006 with all renal controls \\
\hline Cystine $^{*}>4$ & 12 & $3.5-36(10.5)$ & 84-1487 (788) & $<0.001$ with all renal controls \\
\hline Cystinosis patients with ESRD & 5 & $8-24(12)$ & $899-3880$ (1346) & 0.002 with all controls with ESRD \\
\hline Newly diagnosed patients & 9 & $0.8-2.7(1.6)$ & $0-288(122)$ & $<0.001$ with all controls $<5$ years \\
\hline
\end{tabular}

*WBC cystine in nmol 1/2 cystine/mg protein; ESRD, end stage renal disease. 


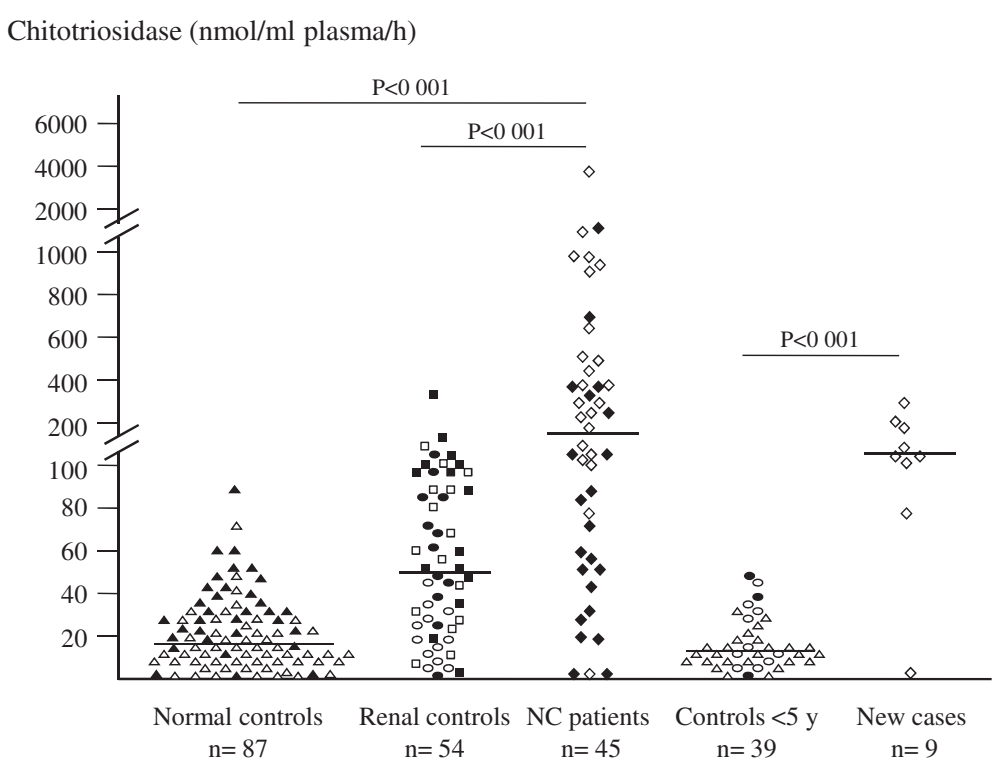

Figure 1 Plasma Chitotriosidase activities in different test groups. Normal pediatric controls $(\Delta)$, normal adult controls $(\boldsymbol{\Delta})$, pediatric controls with renal Fanconi syndrome $(\circ)$, pediatric controls with ESRD $(\bullet)$, adult renal controls with microalbuminuria $(\square)$, adult renal controls with ESRD ( $\mathbf{(})$ and Cystinotic patients; European $(\$)$ and Egyptian ( $(\mathbf{)})$. Forty five cystinotic patients were compared to 54 healthy pediatric controls, 33 healthy adult controls, 24 renal disease pediatric patients (12 with Fanconi syndrome and 12 with ESRD) and 30 renal disease adult patients ( 15 with albuminuria and 15 with ESRD). Nine newly diagnosed Egyptian patients were compared to all controls below 5 years of age ( 26 normal and 13 renal). Averages of duplicates were used. Horizontal bars represent the median value for each group.

22 European patients it ranged from 0.27 to 4.1 (median $1.1 \mathrm{nmol} 1 / 2$ cystine/mg protein), $\mathrm{r}=0.8$ and 0.78 with corresponding chitotriosidase values, respectively. Combined Pearson correlation coefficient was $\mathrm{r}=0.8, \quad \mathrm{P}<0.001$ (Figure 2).

Over the last year we further evaluated a naïve set of suspected patients $(n=13)$ who were referred to our

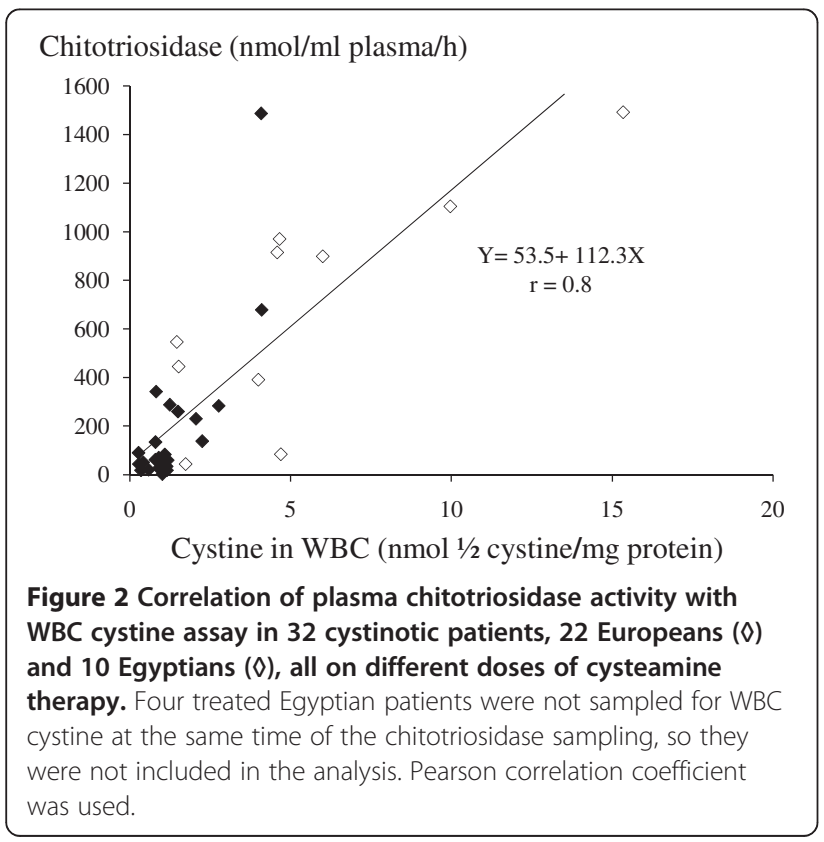

laboratory for WBC cystine assay. WBC cystine levels for nine negative cases ranged from 0.06 to $0.36 \mathrm{nmol} 1 / 2$ cystine/mg protein, while their chitotriosidase activities ranged from 7 to $43 \mathrm{nmol} / \mathrm{ml} / \mathrm{h}$. As for the four positive cases their cystine levels ranged from 3.9 to $12.3 \mathrm{nmol} 1 / 2$ cystine/mg protein, and their chitotriosidase activities ranged from 76 to $373 \mathrm{nmol} / \mathrm{ml} / \mathrm{h}$.

\section{Chitotriosidase genotype}

Thirty six patients were analyzed for the presence of the 24-bp duplication. We found 21 wild-type, 13 heterozygous and two homozygous for the mutation. Three patients were below detection limit for chitotriosidase in our study, two of them were homozygous for the mutation (one Egyptian and one European) and the third (a 2 year's old treated European child) was heterozygous (Figure 1).

\section{Macrophage activation by cystine crystals}

Uptake of cystine crystals by mature macrophages was demonstrated by phase contrast microscopy (Figure 3). Over $95 \%$ of macrophages were still strongly attached to the culture flask surface after 48 hours of crystal elimination denoting cell viability. Looking for direct evidence of macrophage activation after crystal uptake, TNF- $\alpha$ was measured at predetermined intervals. The detected peak level for TNF- $\alpha$ was eight hours after crystal elimination, and the maximum response was obtained with the highest crystal concentration used 


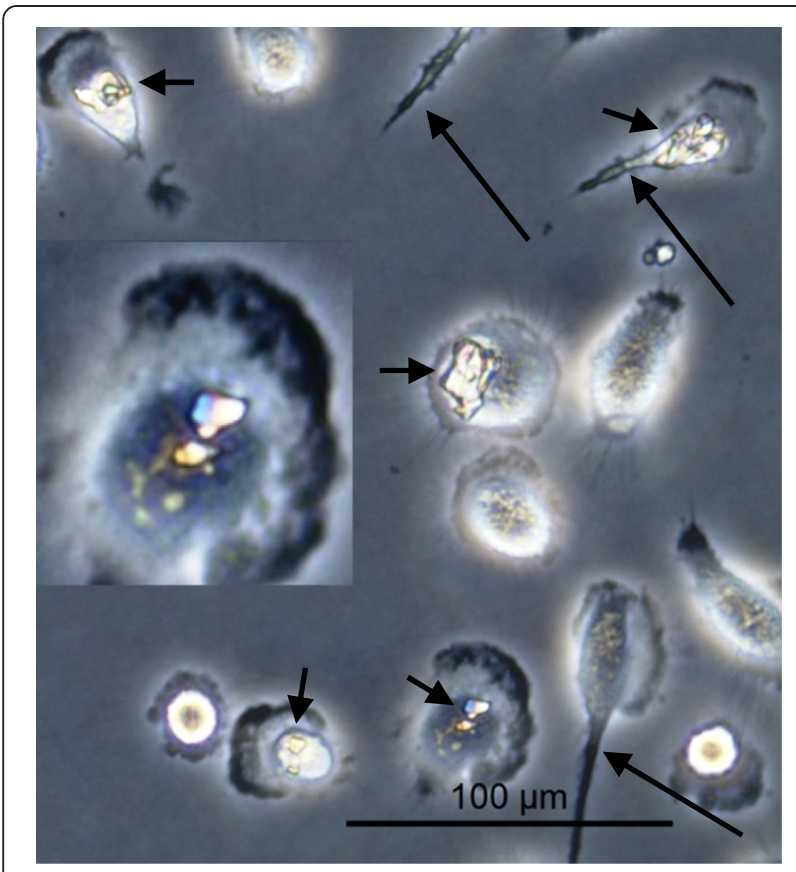

Figure 3 A phase contrast micrograph of macrophages in culture after exposure to cystine crystals. Many macrophages show intracellular crystals (short arrows) and some show pseudopodia (long arrows) to help in movement and engulfment of the crystals.

$\left(200 \mu \mathrm{g} / \mathrm{cm}^{2}\right)$, which was about ten folds the zero level response (Figure 4).

\section{Chitotriosidase activity in macrophage cell culture}

Chitotriosidase activity was increased in the supernatant and cell-lysate of macrophages incubated with cystine crystals (Figure 5A and B). Activity in the supernatant

TNF- $\alpha$ (pg/ug protein)

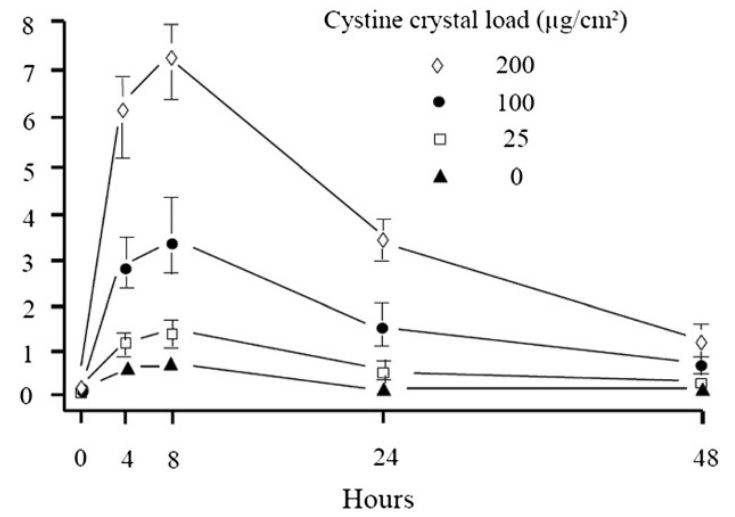

Figure 4 TNF- $a$ concentrations in the supernatant of macrophage cell culture after incubation with different concentrations of cystine crystals (experiment 1 ). A triplicate of each concentration was performed in two separate experiments. Concentrations were expressed as pg/ $\mu \mathrm{g}$ protein of macrophage pellets. Horizontal bars represent the range of TNF-a values for each crystal concentration. was higher than in cell-lysate when referred to pellet's protein content indicating the enzyme rapid extracellular release. Chitotriosidase activities were significantly different from zero level crystal concentration at 48 hours for most concentrations tested, however a trend was observed already after 24 hours.

\section{Chitotriosidase activity in mice}

In an attempt to consolidate our hypothesis in a different species, we found a significant difference between plasma chitotriosidase levels in Ctns(-/-) mice (100-198, median $137 \mathrm{nmol} / \mathrm{ml} / \mathrm{h}, \mathrm{n}=10)$ and $\mathrm{Ctns}(+/+)$ mice $(61-82$, median $73 \mathrm{nmol} / \mathrm{ml} / \mathrm{h}, \mathrm{n}=10), \mathrm{P}=0.003$.

\section{Discussion}

Macrophage activation has been previously reported in many LSDs. Although exact mechanisms are largely unknown, this might reflect the altering signaling pathways in response to storage materials [19]. Furthermore, different types of crystals such as calcium oxalate [18] and monosodium urate [20] were shown to stimulate human macrophages. Tissue macrophages harboring cystine crystals have been demonstrated in cystinotic patients long ago [21,22]. Several immune enhancing molecules such as interferon-gamma, TNF- $\alpha$ and lipopolysaccharide have all been shown to stimulate chitotriosidase expression in human macrophages [23]. We hypothesized that in analogy to other crystal types cystine crystals are not inert structures; they tend to activate macrophages and as a consequence of the elicited activation, chitotriosidase release can be detected clinically and confirmed experimentally.

In our study, apart from a single newly-diagnosed cystinotic patient homozygous for the chitotriosidase deficiency mutation, there was no overlap in chitotriosidase values between the other twelve newly-diagnosed ( 8 in the study group and 4 naivve patients) and age-matched controls (39 in the study group and 9 naïve patients). This indicates that chitotriosidase can be a helpful guide in suspected individuals when WBC cystine measurement is not immediately available, at least to prioritize patients as high or low risk till properly diagnosed.

Many poorly controlled cystinotic patients achieved very high chitotriosidase activities (Figure 1); the highest value $(3880 \mathrm{nmol} / \mathrm{ml} / \mathrm{h}, 216$ folds the median value of normal controls) was observed in an eight years old Egyptian boy with ESRD who died shortly after this measurement. The median chitotriosidase value in the 45 cystinotic patients was about 10 folds the median of normal controls. Apart from Gaucher's disease which has extremely high chitotriosidase values few other LSDs achieve moderately high values comparable to cystinosis, especially sphingolipidoses as Niemann-Pick, GM1 and Krabbe [15,24]. 

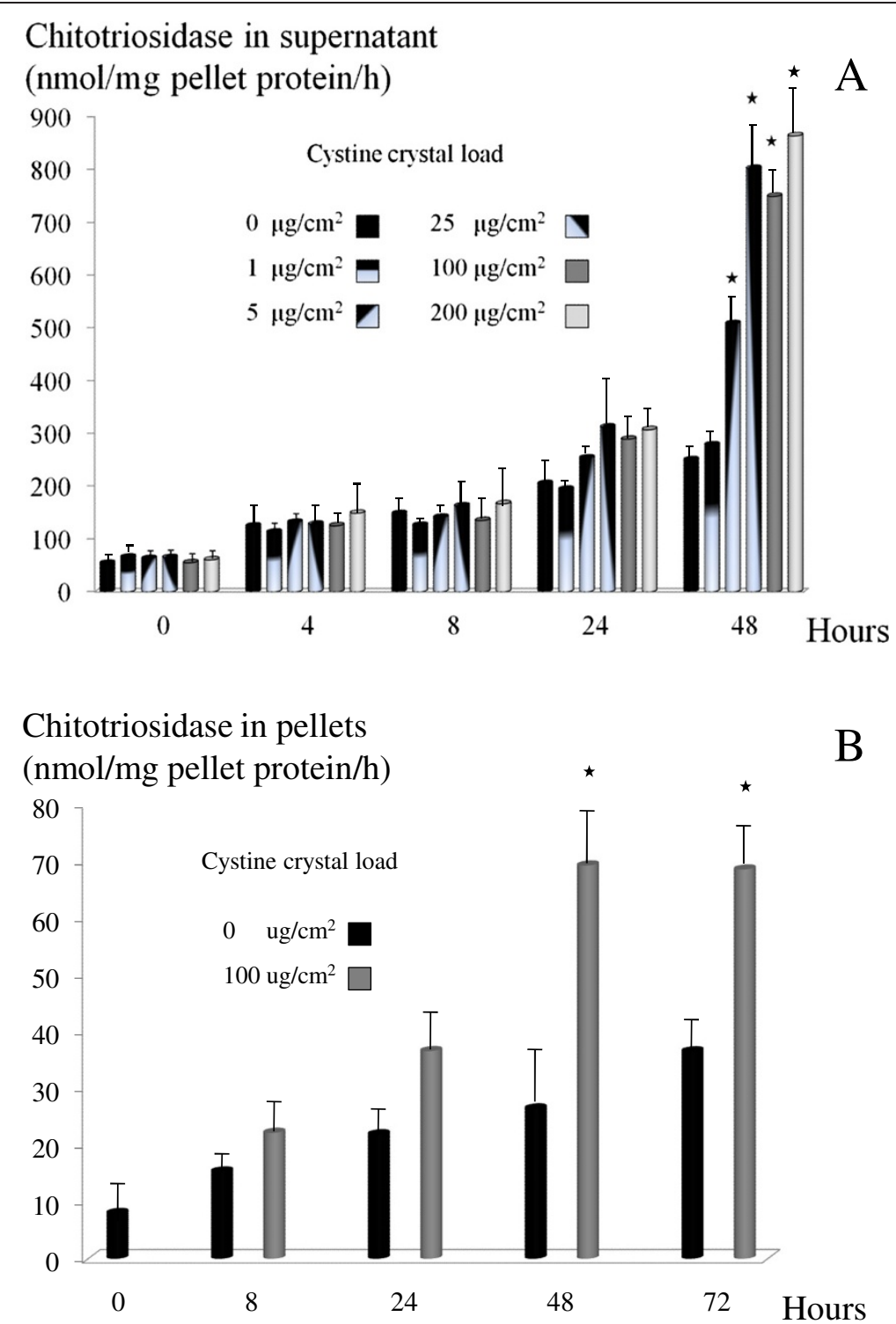

Figure 5 Chitotriosidase activity in the supernatant and cell-lysate of macrophages after incubation with cystine crystals.

(A) Chitotriosidase activity in the supernatant of macrophages incubated with different concentrations of cystine crystals (experiment 1). (B) Chitotriosidase activity in the cell-lysate of macrophages incubated with $100 \mathrm{ug} / \mathrm{cm}^{2}$ cystine crystals (experiment 2). Results were represented as averages and standard deviations of 2 independent experiments in triplicate for experiment 1 and in duplicate for experiment 2 . Enzyme activities for both experiments were expressed as nmol enzyme activity/mg pellet protein/h. ${ }^{*}$ represents $\mathrm{P}<0.05$ with the zero level crystal concentration.

European patients were generally much better therapeutically controlled than Egyptian patients. There was no major difference in the severity of responsible mutations as most mutations were truncating in both cohorts; however, this difference in therapeutic response might be due to the delayed diagnosis and suboptimal therapeutic doses of cysteamine in Egyptian patients given the financial and logistical constraints in providing this orphan drug in a developing country [25]. This was evident from WBC cystine levels in each group. As it could be expected, chitotriosidase activities were much lower in European patients in accordance with the proposed role of chitotriosidase as an alternative therapeutic monitor.

When compared to WBC cystine, plasma chitotriosidase is much easier, faster, more economic, stable and needs a much smaller sample (1 $\mathrm{ml}$ or less) which is more convenient in young children. Furthermore, WBC cystine is not the perfect measure of therapeutic response, as polymorphonuclear leucocytes harboring cystine are short living cells $(\approx 12$ hours); therefore, WBC cystine represents relatively a short time of therapeutic control. Hypothetically, if a patient complies strictly with 
cysteamine treatment a few days before the assay, he may appear properly controlled regardless of previous compliance status. Chitotriosidase, on the other hand, is produced by macrophages which are longer living cells (months to years), and therefore should provide a better notion about therapeutic response over a longer time period. For confirming this statement a more extended longitudinal study is currently underway to better correlate clinical course under cysteamine therapy with chitotriosidase levels.

In newly-diagnosed patients, although chitotriosidase levels were significantly higher than age-matched controls; there was a poor correlation with WBC cystine in cases diagnosed below two years of age. This could be explained by the relative immaturity of human macrophages in infants and young children [26] and better preservation of kidney function, therefore necessitating cautious interpretation of chitotriosidase levels in this age group.

Two of our patients (2/45 or $4.4 \%)$ were homozygous for the 24-bp duplication mutation in accordance with the previously reported prevalence [13]. The third patient having values below the detection limit (A properly controlled 2 years old European child) was heterozygous. The second sample taken few months later showed higher level of chitotriosidase (within the enzyme detectable range) that also corresponded with a higher WBC cystine value.

We have to stress here that chitotriosidase activity will not replace the WBC cystine assay in NC diagnosis; however, we believe that it is a useful clinical screening test and a promising therapeutic monitor. Concerns about false negative screening when the CHIT1 gene is homozygously mutated can be met with setting a lower cut-off limit for the assay, below which the chitotriosidase result is considered of no value and the suspected patient should proceed anyway to WBC cystine assay or to molecular diagnosis.

Although three of our newly diagnosed patients were heterozygous for the CHIT1 mutation, their plasma chitotriosidase activities $(78,122$ and $138 \mathrm{nmol} / \mathrm{ml} / \mathrm{h}$ ) were clearly above their age-matched controls (0-49 nmol/ $\mathrm{ml} / \mathrm{h}, \mathrm{n}=39$ ). Furthermore, recent evidence in Gaucher's disease implies that chitotriosidase activities in the heterozygous patients, although significantly decreased compared to the wild-type, respond to treatment in a very similar way. Chitotriosidase activities in the wild type Gaucher's patients after one year of starting enzyme replacement therapy decreased by $79 \pm 13 \%$ of base line values, while in a similar cohort of heterozygous patients the percent of decrease was also similar $77 \pm 16 \%$ [27]. Likewise, Gaucher patients with the common CHIT1 polymorphism p.G102S were reported to have a similar decline in chitotriosidase activity in response to optimal enzyme replacement therapy $(72 \pm 18 \%$ in the heterozygous and $76 \pm 20 \%$ in the homozygous) [27]

We noticed significant elevations in chitotriosidase activity in control subjects with ESRD compared to normal pediatric and adult controls $(\mathrm{P}<0.001)$. This could be explained by the fact that macrophage infiltration of renal tissue is a common feature of most human chronic kidney disease and that pathogenic M1 subpopulations are implicated in the pathology of renal fibrosis [28]. However, chitotriosidase levels in ESRD controls were still much lower compared to NC patients with the same degree of renal disease because macrophage activation in cystinosis is a global pathology and not just restricted to the kidney.

There are two possible mechanisms of macrophage activation by cystine in cystinotic patients. The first is the gradual accumulation of cystine inside macrophages up to crystallization because macrophages also have cystinosin defect and cannot get rid of their lysosomal cystine similar to other cell types. The second mechanism is the engulfment of dying and apoptotic cells in different tissues with ready formed cystine crystals inside or around these cells and because macrophages have the same pathology they cannot dissolve these phagocytized crystals.

We tried to address both mechanisms initially and used cystine dimethyl ester (CDME) as a rapid loading molecule of cystine into cultured macrophages; however, this molecule was toxic to macrophages and we couldn't achieve our target with non-toxic levels. Using patients macrophages was not feasible because of the high amount of blood required for the experiments. Thus, we addressed the second mechanism through the direct incubation of control human macrophages with different concentrations of cystine crystals in-vitro and succeeded in demonstrating the phagocytosis of cystine crystals, the activation of macrophages only by the crystals and the release of chitotriosidase enzyme.

TNF- $\alpha$, being an established marker of macrophage activation and a direct stimulant of chitotriosidase release [23], was measured sequentially in the supernatant of macrophages exposed to cystine crystals. TNF- $\alpha$ quantified response was correlated with the crystal concentration used (Figure 4), denoting the evident activation of macrophages upon exposure and engulfment of crystals. Macrophage activation was further confirmed through the elevation of chitotriosidase activity in both supernatant and cell-lysate. In fact, this was demonstrated in control macrophages that were capable of processing cystine, while in-vivo this stimulation might be even more pronounced in cystinotic macrophages.

Recently, Principe et al. attributed NC with the inflammasome system activation and argued that the inflammation caused by cystine crystals could be responsible for the 
rapid progression to ESRD in cystinosis unlike other types of hereditary Fanconi syndromes [29]. Similar observation was made by Knauf et al. who demonstrated the importance of the inflammasome mediator NALP3 in the renal injury and fatality of mice with oxalate nephropathy [30]. Okamura et al. further elucidated the role of cysteamine treatment in reducing macrophage accumulation in the renal interstitium of mice exposed to chronic renal injury and reducing the generation of reactive oxygen species in these macrophages [31]. Thus, the clinical assessment of macrophage activation in $\mathrm{NC}$ as an indicator of disease activity and/or therapeutic response seems to be a promising approach. Measuring chitotriosidase activity in that regard has many advantages compared to other circulating cytokines, especially that the enzyme is long-living and very stable, thus, less affected by acute infections or immunosuppressive drugs and it can be also measured in blood spots [32].

The significant elevation of plasma chitotriosidase in Ctns(-/-) mice indicates that its correlation with cystinosis is not species specific. Although the median value for the diseased was only double that of the wild-type, this might refer to the shorter duration of the pathological process and the relatively lower differential number of activated macrophages in mice [33].

The recent successful treatment of the murine cystinotic model by bone marrow cell transplantation [34] and hematopoietic stem cell gene therapy [35], if proven successful in human patients, will have a powerful impact on the disease therapeutic strategy. However, the problem on how to evaluate the patient's response posttransplantation is not yet solved as estimating cystine accumulation in tissue biopsies is too invasive for routine follow-up and measuring cystine in the donor's WBCs is useless. Chitotriosidase, on the other hand, is rapid and non-invasive. When compared to a base-line level it would represent the activity of the recipient's tissue macrophages and would be a reasonable indicator of total body cystine load. Notably, chitotriosidase enzyme was reported to show significant declines in response to successful bone marrow transplantation in Gaucher's disease [14] and in $\beta$-thalassemia major [36].

\section{Conclusions}

In conclusion, plasma chitotriosidase activity was significantly elevated in cystinotic patients at diagnosis over age matched normal and renal controls and correlated with WBC cystine assay for treated patients above 2 years of age. Thus, chitotriosidase is a promising clinical biomarker and therapeutic monitor in nephropathic cystinosis patients.

\section{Competing interests}

The authors declare that they have no competing interests.

\section{Authors' contributions}

MAE performed the experimental studies and drafted the manuscript. LVH, NAS, and EL designed the experiments, standardized the protocols and drafted the manuscript. SHM, HA, SMA, MMN, FAH and NAS were responsible for collecting data of Egyptian cystinotic patients. MCJ, EAMC, $D J L, L A B J$ and $E L$ were responsible for collecting data of European cystinotic patients. XB provided theoretical and technical support for macrophage experiments. HPG and FOA provided theoretical and technical support for animal experiments. All the authors have revised the manuscript critically and approved its submission.

\section{Acknowledgements}

The authors sincerely thank the affected patients and their families for participation. EL is supported by the fund for Scientific Research, Flanders (F.W.O. Vlaanderen) grant $1801110 \mathrm{~N}$ and by the Cystinosis Research Foundation (CRF). The Egyptian Group of Orphan Renal Diseases (EGORD) funded laboratory investigations and cysteamine eye drops and oral treatment for Egyptian patients. MAE was supported by the Cystinosis Research Network (CRN). We gratefully acknowledge Prof. Dr. Fatma El-Mougy for her kind and persistent support, Prof. Dr. Corinne Antignac and Prof. Dr. Pierre Courtoy for their constructive comments and for supplying mice plasma samples and we would like also to thank Inge Bongaers, Sandra Van Aerschot and Greet Wuyts for valuable technical assistance. Aimé Van Gucht is acknowledged for revising English language and grammar.

\section{Author details}

${ }^{1}$ Department of Clinical and Chemical Pathology, Inherited Metabolic Disorder Laboratory (IMDL), Cairo University, Cairo, Egypt. ²Department of Pediatrics, Center of Pediatric Nephrology and Transplantation (CPNT), Cairo University, Cairo, Egypt. ${ }^{3}$ EGORD, Egyptian group of orphan renal diseases, Cairo, Egypt. ${ }^{4}$ Department of Pediatric Nephrology \& Growth and Regeneration, University Hospital Leuven, Catholic University of Leuven, Leuven, Belgium. ${ }^{5}$ Department of Pediatric Nephrology, Radboud University Medical Center, Nijmegen, The Netherlands. ${ }^{6}$ University Hospitals Leuven \& Department of Microbiology and Immunology, Laboratory Medicine, Catholic University of Leuven, Leuven, Belgium. 'Department of Internal Medicine, Radboud University Medical Center, Nijmegen, The Netherlands.

${ }^{8}$ Department of Neurology, Laboratory for Genetic, Endocrine and Metabolic Diseases, Radboud University Medical Center, Nijmegen, The Netherlands.

${ }^{9} \mathrm{CELL}$ Unit, Université Catholique de Louvain \& de Duve Institute, Bruxelles, Belgium.

Received: 29 July 2014 Accepted: 30 September 2014

Published online: 19 November 2014

\section{References}

1. Nesterova G, Gahl WA: Cystinosis: the evolution of a treatable disease Pediatr Nephrol 2013, 28:51-59.

2. Jézégou A, Llinares E, Anne C, Kieffer-Jaquinod C, O'Regan S, Aupetit J, Chabli A, Sagné C, Debacker C, Chadefaux-Vekemans B, Journet A, André B, Gasnier B: Heptahelical protein PQLC2 is a lysosomal cationic amino acid exporter underlying the action of cysteamine in cystinosis therapy. Proc Natl Acad Sci U S A 2012, 109:E3434-E3443.

3. Wilmer MJ, Schoeber JP, van den Heuvel LP, Levtchenko EN: Cystinosis: practical tools for diagnosis and treatment. Pediatr Nephrol 2011, 26:205-215.

4. Oshima RG, Willis RC, Furlong CE, Schneider JA: Binding assays for amino acids. The utilization of a cystine binding protein from Escherichia coli for the determination of acid-soluble cystine in small physiological samples. J Biol Chem 1974, 249:6033-6039.

5. de Graaf-Hess A, Trijbels F, Blom H: New method for determining cystine in leukocytes and fibroblasts. Clin Chem 1999, 45:2224-2228.

6. Chabli A, Aupetit J, Raehm M, Ricquier D, Chadefaux-Vekemans B: Measurement of cystine in granulocytes using liquid chromatographytandem mass spectrometry. Clin Biochem 2007, 40:692-698.

7. Levtchenko E, de Graaf-Hess A, Wilmer M, van den Heuvel L, Monnens L, Blom H: Comparison of cystine determination in mixed leukocytes vs polymorphonuclear leukocytes for diagnosis of cystinosis and monitoring of cysteamine therapy. Clin Chem 2004, 50:1686-1688.

8. Hollak CE, van Weely S, van Oers MH, Aerts JM: Marked elevation of plasma chitotriosidase activity. A novel hallmark of Gaucher disease. J Clin Invest 1994, 93:1288-1292. 
9. Guo Y, He W, Boer AM, Wevers RA, de Bruijn AM, Groener JE, Hollak CE, Aerts JM, Galjaard H, van Diggelen OP: Elevated plasma chitotriosidase activity in various lysosomal storage disorders. J Inherit Metab Dis 1995, 18:717-722.

10. Barone R, Di Gregorio F, Romeo MA, Schilirò G, Pavone L: Plasma chitotriosidase activity in patients with beta-thalassemia. Blood Cells Mol Dis 1999, 25:1-8.

11. Boot RG, Hollak CE, Verhoek M, Alberts C, Jonkers RE, Aerts JM: Plasma chitotriosidase and CCL18 as surrogate markers for granulomatous macrophages in sarcoidosis. Clin Chim Acta 2010, 411:31-36.

12. van Eijk M, van Roomen CP, Renkema GH, Bussink AP, Andrews L, Blommaart EF, Sugar A, Verhoeven AJ, Boot RG, Aerts JM: Characterization of human phagocyte-derived chitotriosidase, a component of innate immunity. Int Immunol 2005, 17:1505-1512.

13. Boot RG, Renkema GH, Verhoek M, Strijland A, Bliek J, de Meulemeester TM, Mannens MM, Aerts JM: The human chitotriosidase gene. Nature of inherited enzyme deficiency. J Biol Chem 1998, 273:25680-25685

14. Young E, Chatterton C, Vellodi A, Winchester B: Plasma chitotriosidase activity in Gaucher's disease patients who have been treated either by bone marrow transplantation or by enzyme replacement therapy with alglucerase. J Inherit Metab Dis 1997, 20:595-602.

15. Ries M, Schaefer E, Lührs T, Mani L, Kuhn J, Vanier MT, Krummenauer F, Gal A, Beck M, Mengel E: Critical assessment of chitotriosidase analysis in the rational laboratory diagnosis of children with Gaucher disease and Niemann-Pick disease type A/B and C. J Inherit Metab Dis 2006, 29:647-652.

16. Vedder AC, Cox-Brinkman J, Hollak CE, Linthorst GE, Groener JEM, Helmond MTJ, Aerts JM: Plasma chitotriosidase in male Fabry patients: a marker for monitoring lipid-laden macrophages and their correction by enzyme replacement therapy. Mol Genet Metab 2006, 89:239-244.

17. Xaidara A, Karavitakis EM, Kosma K, Emma F, Dimitriou E, Michelakakis H: Chitotriosidase plasma activity in nephropathic cystinosis. J Inherit Metab Dis 2009, 32(Suppl):157-159.

18. de Water R, Leenen PJ, Noordermeer C, Nigg AL, Houtsmuller AB, Kok DJ, Schröder $\mathrm{FH}$ : Cytokine production induced by binding and processing of calcium oxalate crystals in cultured macrophages. Am J Kidney Dis 2001, 38:331-338

19. Vitner EB, Platt FM, Futerman $\mathrm{AH}$ : Common and uncommon pathogenic cascades in lysosomal storage diseases. J Biol Chem 2010, 285:20423-20427.

20. Cronstein BN, Sunkureddi P: Mechanistic aspects of inflammation and clinical management of inflammation in acute gouty arthritis. J Clin Rheumatol 2013, 19:19-29.

21. Scotto JM, Stralin HG: Ultrastructure of the liver in a case of childhood cystinosis. Virchows Arch A Pathol Anat Histol 1977, 377:43-48.

22. lancu TC, Lerner A, Shiloh H: Intestinal mucosa in nephropathic cystinosis. J Pediatr Gastroenterol Nutr 1987, 6:359-364.

23. Malaguarnera L, Musumeci M, Di Rosa M, Scuto A, Musumeci S: Interferon-gamma, tumor necrosis factor-alpha, and lipopolysaccharide promote chitotriosidase gene expression in human macrophages. J Clin Lab Anal 2005, 19:128-132.

24. Wajner A, Michelin K, Burin MG, Pires RF, Pereira ML, Giugliani R, Coelho JC: Comparison between the biochemical properties of plasma chitotriosidase from normal individuals and from patients with Gaucher disease, GM1-gangliosidosis, Krabbe disease and heterozygotes for Gaucher disease. Clin Biochem 2007, 40:365-369.

25. Soliman NA, Elmonem MA, van den Heuvel L, Hamid RH, Gamal M, Bongaers I, Marie S, Levtchenko E: Mutational Spectrum of the CTNS Gene in Egyptian Patients with Nephropathic Cystinosis. JIMD Rep 2013, doi:10.1007/8904_2013_288.

26. Grigg J, Riedler J, Robertson CF, Boyle W, Uren S: Alveolar macrophage immaturity in infants and young children. Eur Respir J 1999, 14:1198-1205.

27. Irún P, Alfonso P, Aznarez S, Giraldo P, Pocovi M: Chitotriosidase variants in patients with Gaucher disease. Implications for diagnosis and therapeutic monitoring. Clin Biochem 2013, 46:1804-1807.

28. Wang Y, Harris DCH: Macrophages and renal disease. J Am Soc Nephrol 2011, 22:21-27.

29. Prencipe G, Caiello I, Cherqui S, Whisenant T, Petrini S, Emma F, De Benedetti F: Inflammasome Activation by Cystine Crystals: Implications for the Pathogenesis of Cystinosis. J Am Soc Nephrol 2014, 25:1163-1169.

30. Knauf F, Asplin JR, Granja I, Schmidt IM, Moeckel GW, David RJ, Flavell RA, Aronson PS: NALP3- mediated inflammation is a principal cause of progressive renal failure in oxalate nephopathy. Kidney Int 2013, 84:895-901.
31. Okamura DM, Bahrami NM, Ren S, Pasichnyk K, Williams JM, Gangoiti JA, Lopez-Guisa JM, Yamaguchi I, Barshop BA, Duffield JS, Eddy AA: Cysteamine modulates oxidative stress and blocks myofibroblast activity in CKD. J Am Soc Nephrol 2014, 25:43-54.

32. Elmonem MA, Ramadan DI, Issac MS, Selim LA, Elkateb SM: Blood spot versus plasma chitotriosidase: A systematic clinical comparison. Clin Biochem 2014, 47:38-43.

33. Nevo N, Chol M, Bailleux A, Kalatzis V, Morisset L, Devuyst O, Gubler MC, Antignac C: Renal phenotype of the cystinosis mouse model is dependent upon genetic background. Nephrol Dial Transplant 2010, 25:1059-1066.

34. Syres K, Harrison F, Tadlock M, Jester JV, Simpson J, Roy S, Salomon DR, Cherqui S: Successful treatment of the murine model of cystinosis using bone marrow cell transplantation. Blood 2009, 114:2542-2552.

35. Harrison F, Yeagy BA, Rocca CJ, Kohn DB, Salomon DR, Cherqui S: Hematopoietic stem cell gene therapy for the multisystemic lysosomal storage disorder cystinosis. Mol Ther 2013, 21:433-444.

36. Maccarone C, Pizzarelli G, Barone R, Musumeci S: Plasma chitotriosidase activity is a marker of recovery in transplanted patients affected by beta-thalassemia major. Acta Haematol 2001, 105:109-110.

doi:10.1186/s13023-014-0155-z

Cite this article as: Elmonem et al:: Clinical utility of chitotriosidase enzyme activity in nephropathic cystinosis. Orphanet Journal of Rare Diseases 2014 9:155.

\section{Submit your next manuscript to BioMed Central and take full advantage of:}

- Convenient online submission

- Thorough peer review

- No space constraints or color figure charges

- Immediate publication on acceptance

- Inclusion in PubMed, CAS, Scopus and Google Scholar

- Research which is freely available for redistribution 